\author{
Marie Giraud-Claude-Lafontaine \\ Jagiellonian University \\ ul. Gołębia 24, \\ 31-007 Cracow, Poland
}

\title{
La neige et la guerre \\ dans La neige était sale de Georges Simenon et Warna ou le poids de la neige de Paul Willems
}

\begin{abstract}
The purpose of this article entitled "The snow and the war in The Snow was dirty by Georges Simenon and Warna or the weight of the snow by Paul Willems" is to show how the snow is playing a structuring role in the two studied works: first, due to its ambivalent properties, it makes the plot switch, secondly, it lets overcome the reality of the war in order to explore general questions as fate, purity and redemption.

Keywords: snow; war; Second World War; memory; fate; purity; redemption
\end{abstract}

Il est tentant d'associer l'image de la neige à la guerre : flaques de sang sur fond immaculé, froid aggravant les souffrances, chiens affamés menaçant les enfants mal nourris... Esthétisme cliché et symbolique éprouvée ? Certes, si l'on se contente d'un décorum éculé, mais lorsque la neige est au cœur du mécanisme narratif ou dramatique et qu'elle donne une «matière » à l'imagerie poétique pour paraphraser Bachelard (1942: 5), il en va tout autrement. Neige pesante dans Warna, neige crasseuse dans La neige était sale, la neige, 
bien que moins mobile et fuyante que l'eau, est aussi changeante, raison pour laquelle on peut affirmer que son image matérielle s'associe comme l'eau au destin (Bachelard 1942: 8). Si «la mort quotidienne est la mort de l'eau » et que «la peine de l'eau est infinie » (Bachelard 1942: 9), que dire de la neige qui emprisonne le temps d'une saison l'eau qui la compose, donnant l'illusion d'un destin qui s'arrête et d'une mort neutralisée ? Car la question de la mort, associée à la guerre et liée à la neige, est au cœur de l'étude de nos deux œuvres. Curieusement, dans les deux cas, le mot neige a été ajouté après coup : le roman de Georges Simenon, écrit en mars 1948, quelques mois après la confirmation officielle de la mort de son frère Christian engagé dans la Légion étrangère après s'être tristement rendu célèbre lors de la tuerie de Courcelles, était initialement intitulé Mr. Host. Quant à Paul Willems, il remanie en 1984 sa pièce Warna (1963) et la dote du sous-titre éloquent: ou le poids de la neige. Le but de notre analyse sera de montrer comment la neige est un moteur dramatique/narratif qui dépasse les incertitudes référentielles et compose une poétique conjurant les effets de la guerre, et ce, dans deux œuvres fort dissemblables tant du point de vue du genre que du ton et du style.

1. Comment dire la guerre: une guerre indéterminée et transposée dans le quotidien

«Ostende pendant la guerre. Quelle guerre ? N'importe quelle guerre. Oublier l'histoire, l'occupation. Retenir le vide. Ostende, isolée, n'est plus que le tombeau des saisons révolues »(Willems 1974 : 9). Dans les deux œuvres, il est difficile de situer la guerre, de manière encore plus évidente dans Warna, puisqu'on est dans un univers imaginaire déclaré (Willems 1984a: 8), mais aussi dans La neige était sale, roman de type pourtant réaliste. Dans Warna, la guerre est une menace lointaine : le château est protégé des pillages et des incendies par les étangs. Les références à un contexte historique sont nombreuses mais imprécises : elles évoquent soit des conflits indéterminés entre les Français et les «Impériaux » $(W$, p. 20, 14), soit les guerres de religion du XVI ${ }^{\mathrm{e}}$ siècle $(W, \mathrm{p} .15,20)$. Nous sommes en 
Flandre, Flandre mythique, terre marécageuse, champ de bataille des deux puissances voisines. L'intrigue de La neige était sale se situe clairement au milieu du $\mathrm{XX}^{\mathrm{e}}$ siècle, dans le monde occidental. Le roman ayant été écrit trois ans après la fin de la Seconde guerre mondiale, même si les occupants ne sont pas caractérisés - on sait seulement qu'ils sont étrangers - tout porte à croire que l'histoire se passe sous l'occupation nazie, en Belgique ou dans le nord de la France. Pourtant, comme Simenon l'a écrit à Frédéric Dard, rien n'est moins certain :

Dans mon livre, il n'est jamais question d'Allemands. Je voudrais en effet que l'occupant soit aussi neutre que possible afin de donner plus de généralité à l'œuvre. À vrai dire, dans mon esprit, l'action se passe en Europe centrale, et plutôt sous l'occupation russe (Assouline 1992 : 559).

En effet, les occupés ont des noms à consonance germanique : Kromer, Bertha, Friedmaier, Kurt Hamling, plutôt allemands que flamands. Dans les deux œuvres, il se passe donc une «dilatation » des faits historiques où le réel de la guerre n'est pas nié mais « diffracté » dans la fiction (Quaghebeur 2017 : 41), ce qui correspond aussi à l'expérience de la guerre des deux auteurs: Willems l'a vécue de manière irréelle pendant la campagne des 18 jours (Willems 1984b: 202-203) et Simenon l'a fuie, se terrant toujours plus profondément dans la campagne vendéenne (Assouline $1992: 409$ ).

Autre caractéristique commune aux deux œuvres, dans cette guerre d'usure, dont on ne sait situer le début ni envisager la fin, il n'est pas question des combats du front: les conflits sont transposés dans la sphère quotidienne, opposant les privilégiés et les défavorisés. Dans Warna, les habitants du château sont à l'abri du froid et de la guerre et croulent sous l'abondance ( $W, \mathrm{p} .14)$ alors que les habitants de l'auberge subissent la férocité des soldats, le froid, et la faim ( $W, \mathrm{p}$. 15) ; dans La neige, les collaborateurs sont gavés de charbon, de café, de copieux repas, d'alcool, de vêtements chauds, tandis que les autres subissent une restriction sévère. Ces privilèges, Simenon y a goûté aussi : sans pour autant tomber dans l'abjection d'une collaboration active comme son frère, il a sans conteste mieux vécu l'Occupation que la Libération. Quant à Willems, si c'est une période pendant 
laquelle il s'est marié et s'est senti socialement utile, il a eu le malheur de perdre son frère dans un bombardement. En ce qui le concerne, même si rien ne le prouve, on pourrait se demander si, plutôt qu'à sa propre expérience, il ne se réfère pas davantage au roi des Belges, enfermé dans sa prison dorée de Laeken, ne boudant pas le plaisir d'un remariage illégal.

Dans les deux cas, la guerre devient une lutte interne : dans Warna, le conflit est déclaré entre le château et l'auberge puis entre les personnages construits en miroir: le chevalier Ernevelde et l'aubergiste, la vieille Marthe et Warna. Ernevelde se débarrasse de son rival, Malo, en faisant croire à une attaque ennemie ( $W$, p. 86). La guerre devient le prétexte à des règlements de compte personnels, faisant pénétrer le crime dans la sphère privée. Dans La neige, les crimes commis ne sont pas le fruit de combats guerriers et ne résultent pas comme dans Warna de rivalités entre les personnages. Ce sont des meurtres parfaitement gratuits, dénués de motivation sentimentale, qui témoignent d'un cynisme abject et d'un profond mépris pour la vie d'autrui. Dans le sillage de son compagnon Kromer, Frank tue un officier étranger pour se faire la main, sans aucune prétention patriotique. Le dépucelage orchestré de Sissy, une jeune voisine amoureuse de Frank, pousse loin l'abjection : Frank demande à Kromer de se faire passer pour lui, profitant de l'obscurité de la chambre. Déclarant une guerre personnelle à tous les personnages, les poursuivant de son mépris, refusant leur compréhension et leur aide, Frank s'isole des autres et persévère dans l'amoralité.

Ainsi, si l'on peut trouver des éléments dans les deux œuvres qui feraient écho à l'expérience de l'Occupation, les schémas narratif et dramatique ne se satisfont pas de motivations réalistes : structurés en fonction des métamorphoses de la neige, ils opèrent une « diffraction » du réel.

2. Le rôle ambivalent de la neige

- Warna: légèreté et poids de la neige, une dramaturgie poétique de la neige 
Dans la pièce de théâtre, la neige a deux rôles ambivalents : légère pour Warna et Visius, elle est un poids pour les autres personnages, jusqu'à ce que Warna se transforme en statue de la neige, ce qui lui confère le pouvoir de transfigurer le mensonge en délivrance collective. Cette ambivalence tient à la matière même de la neige, légèreté du flocon et poids de la neige : «chaque flocon sur chaque aiguille de sapin se pose en un contact exquis [...] Les branches ploient sous un poids exquis...» $(W$, p. 29). La neige est si présente dans le discours des personnages qu'on a l'impression qu'elle tombe tout au long de la pièce. Or, il n'en est rien : pas un flocon jusqu'au quatrième acte. Au premier acte, les personnages sont réunis dans l'auberge délabrée pour célébrer les vingt-cinq ans d'amour entre Warna et Ernevelde, qui se sont rencontrés le jour de la fête de la neige, raison pour laquelle la neige reste le symbole intact de leur amour. L'idée de la neige est convoquée pour ranimer le souvenir de cet amour éteint et le faire vibrer de nouveau, immobilisant le temps. Malgré l'évidence de la réalité et la misère causée par la guerre, la magie de la neige opère pendant les trois premiers actes. C'est le rôle de Visius, l'écrivain, de rétablir l'illusion par la force de la poésie, par exemple pendant la pitoyable parodie de célébration à l'auberge : «Imaginez-vous que vous vous promenez par un chemin couvert de neige. Une neige merveilleusement légère. Immaculée $»(W$, p. 21$)$. En revanche, ce souvenir poudré est vécu comme un poids par les autres personnages, immobilisés dans le rêve de Warna : Ernevelde est devenu gros, gâté par d'excessifs soins ( $W$, p. 76). Marthe porte en elle une pierre noire ( $W, \mathrm{p} .97)$, un poids d'une douleur infinie $(W, \mathrm{p}$. 103). Or, Warna a besoin des autres pour faire exister son rêve et pour maintenir l'illusion: "il faut des millions de témoins pour qu'une chose existe » dit Marie ( $W$, p. 67). Seul Visius comprend que le château de neige de Warna est une protection contre la réalité : " votre rêve est la seule réalité et nous en vivons... tout le reste est cauchemar»( $W$, p. 82). Maintenir coûte que coûte l'illusion de l'amour revient à éloigner la guerre du château. Certains personnages résistent à la tyrannie du rêve enneigé de Warna, comme Ernevelde qui lui oppose la chaleur orientale d'Ispahan ( $W$, p. 71$)$ et qui s'enfuira 
avec Marie en Bourgogne. L'assassinat de Malo à la fin du troisième acte fait basculer le drame : à l'acte suivant, la neige est tombée. C'est l'occasion d'une première délivrance qui échoue : la pendaison des habitants du château aurait pu libérer les habitants de l'auberge mais les manœuvres d'Ernevelde viennent à bout de l'aubergiste. Le moment de la délivrance est repoussé, jusqu'au moment où la neige commence à tomber, cette fois, à l'intérieur du château. Le poids réel de la neige, associé à la fuite d'Ernevelde est d'abord une légèreté retrouvée: «j'avais l'impression que la neige avait suspendu sa chute et que c'était moi qui m'élevais vers le ciel » $(W$, p. 114). Puis c'est la lourdeur de l'attente, pendant deux mois: la neige a pénétré à l'intérieur du château et avec elle, la réalité rattrape chaque personnage, les mots gèlent ( $W$, p. 124), même Visius se décourage. Sous l'effet de la neige, Warna, ne parvenant plus à immobiliser le temps, s'immobilise elle-même en se métamorphosant en statue de la neige ( $W$, p. 126). Lorsqu'Ernevelde revient, il est lui-même transformé car Marie lui a fait connaitre le véritable amour puis l'abandon. Il est incapable de retourner dans le rêve de Warna. Celleci, pour sauver son illusion, n'a le choix que de le faire disparaître. Une fois qu'il est tué, il se met à neiger, une nouvelle neige légère, la neige de l'ultime délivrance : «C'est étrange... je suis délivré d'un grand poids... $(W$, p. 138) constate Visius. La légèreté de la neige, jusque là réservée principalement à Warna, est alors partagée entre tous.

- La Neige était sale : saleté et pureté, la moralité de la neige en tant que schème narratif

Dans ce roman, la neige joue aussi un rôle ambivalent : tout au long de la première partie, où Frank pousse le cynisme aussi loin qu'il le peut, la neige est tombée il y a longtemps déjà : elle est dure et sale ; juste avant son arrestation, avant la dernière partie du roman consacrée à son emprisonnement, la neige tombe fraîche, blanche et pure.

Dans la première partie du roman, souvent associée à la nuit ou à un ciel gris et menaçant, la neige accompagne chaque crime. Lors de 
son premier meurtre, Frank est embusqué dans une impasse qui n'a pas été déneigée : il a «jusqu'aux genoux les jambes dans la neige durcie » (NS, p. 15), «sur les trottoirs, la neige formait deux banquettes grisâtres »(NS, p. 15). Quand Frank commence à fréquenter la jeune et pure Sissy, une fine neige se met à tomber poétiquement: «des brins de neige viennent fondre sur ses lèvres » (NS, p. 82). Elle est hélas trop fine pour recouvrir l'ancienne et ramener Frank à de meilleurs sentiments :

Il neige toujours fin. C'est comme pour les orages d'été : quand cela dure trop longtemps, on finit par guetter avec angoisse la bonne chute de neige épaisse qui purgera le ciel et permettra de revoir le soleil (NS, p. 82).

Cette neige purificatrice se fait attendre. Alors, Frank persiste dans sa cruauté et se convainc de faire dépuceler sa petite amie par procuration :

Toujours la neige sale, les tas de neige qu'on dirait pourrie, avec des traces noires, des incrustations de détritus. La poudre blanche qui se décolle parfois de la croûte du ciel, par petits paquets, comme le plâtre d'un plafond, ne parvient pas à recouvrir cette crasse (NS, p. 92).

C'est le moment du roman où la neige est la plus sale, à l'image de l'âme de Frank. Enfin, après une nuit de veille à attendre des nouvelles de Sissy, qui a passé la moitié de la nuit dehors, la neige arrive enfin : «La neige tombe, épaisse, silencieuse, si épaisse qu'on distingue à peine les fenêtres de la maison d'en face [...] la qualité de l'air est changée. » (NS, p. 119). À ce moment-là, le roman bascule : Frank a touché le fond de l'abîme de son cynisme. Il commence à s'inquiéter des conséquences de ses actes mais au fond de lui, il attend le châtiment comme un achèvement. Le jour de son arrestation, pendant le trajet qui le conduit à sa prison, Frank est frappé par l'éclat de la ville : «le ciel était brillant, on avait l'impression, ce matin-là, que la ville scintillait de tous ses carreaux, de toute sa neige, de tous ses toits blancs » $(N S$, p. 149). La neige n'a plus sa croûte dure : le mégot de sa cigarette est «comme pompée par l'humidité de la neige au soleil » (NS, p. 149). Il semble bien que la neige soit suffisamment épaisse pour recouvrir l'ancienne saleté mais aussi assez tendre pour 
faire aussitôt disparaitre les nouvelles ordures. Pendant tout son emprisonnement, le ciel restera lumineux et la neige éclatante jusqu'au bout. Il est fusillé le matin où il se remet à neiger.

Dans ce roman, la neige est associée à la notion morale de purification, bien plus nettement que dans Warna, qui, au-delà ou en deçà de toute morale, a une fonction mémorielle: immobiliser le souvenir dans l'imagination puis le faire renaître, ce qui ouvre à une poétique double de la neige, en tant que conjuration de la guerre, c'est-à-dire de la mort.

3. La neige au-delà de la mort : une conjuration de la guerre Dans les deux textes, la guerre est associée à l'idée du manque et de la perte. Cette perte est intériorisée chez Willems et acceptée chez Simenon. Si l'on reprend les deux axes de l'imagination de Bachelard, l'imagination matérielle et l'imagination formelle, on peut considérer que Warna et La neige produisent une poétique complémentaire qui permet d'approcher la perte, c'est-à-dire la mort, en tant que retour aux origines chez Willems (axe vertical) et en tant que destin chez Simenon (axe horizontal). Willems, dans sa «Lettre aux comédiens qui interpréteront Warna », explique qu'en regardant furtivement le spectacle de la neige tombant sur la ville pétrifiée par la guerre, il a compris que la menace «est toujours là, en permanence, au fond de nous-mêmes »(Willems 1984a : 7). La neige se fait alors métaphore de la part enfouie de la perte, au plus profond de nous, qui, lorsqu'elle est atteinte, se manifeste confusément comme « une mémoire d'avant [la] naissance, un songe d'après [la] mort » (Willems 1984a: 8). En revanche, la poétique de La neige était sale suit davantage l'axe horizontal, celui de l'épanouissement de la forme : l'accomplissement $\mathrm{du}$ destin et la rédemption de Frank sont conditionnés par l'acceptation d'une métamorphose morale, opérante grâce à une métamorphose de la neige.

Dans Warna, l'intériorisation de la perte procède d'abord d'un mécanisme d'introduction des éléments naturels extérieurs dans l'espace intérieur : ce sont les vêtements de la châtelaine, les motifs de sa cape vert sombre «qui rappellent des branches de sapin» $(W, \mathrm{p}$. 
42), ou son autre cape « doublée de lièvre des neiges » ( $W$, p. 125). La porosité de la frontière entre l'extérieur et l'intérieur se retrouve dans la rencontre amoureuse, associée chez Willems à la communion avec la nature, en tant que fragment du paradis perdu (Michaux 1988 : 133) : Ernevelde paraît à Warna derrière des gouttes d'eau suspendues aux aiguilles de sapin, les cheveux brillant de pluie et un marcassin tué sur l'épaule ( $W, \mathrm{p}$. 43). Dans la lettre que Warna dicte à Visius pour le chevalier, l'écrivain remplace les mots d'amour par les mots de la nature ( $W$, p. 50). Enfin, le cadavre d'Ernevelde est destiné à être englouti au dégel $(W$, p. 139). Les personnages délivrés à la fin de la pièce sont ceux qui ont expérimenté la perte mais qui en gardent une trace. Sont donc exclus Malo, mort trop tôt, Marie qui secoue son destin et réalise son rêve et l'aubergiste désespérément vide de tout rêve. Au contraire, Gérard a perdu le rêve de bonheur de son enfant mais garde en mémoire son sourire, Warna a perdu son amour mais en maintient l'illusion, Marthe a perdu Ernevelde mais porte un rêve de vengeance, Visius a perdu le souvenir des livres mais a le pouvoir des mots, Anita a perdu sa jeunesse mais possède la beauté du chant, Rémi a perdu la parole mais garde le souvenir du paradis. Une fois la perte assumée, la délivrance s'accomplie et les personnages se rassemblent librement autour de Warna : l'harmonie originelle est retrouvée et les rivalités disparaissent. Sont-ils morts ? Sont-ils au paradis ? Ils sont au seuil du mystère des origines qui n'apparaît clairement qu'à l'ange Rémi qui retrouve la parole. Pour les autres, l'incertitude perdure, car le seuil est atteint au prix de l'annulation de l'Autre (la mort d'Ernevelde) et du retour du même, le retour à la pureté originelle est impossible.

Dans La neige était sale, la persévérance inexpliquée de Frank dans l'abjection a pour origine une provocation du destin. Embusqué pour assassiner l'officier, il a toussé pour attirer l'attention de son voisin, M. Holst, qui passait à ce moment-là et qui ne pouvait manquer de conclure qu'il est l'auteur du crime. Dès lors, « cela aussi et tout ce qui devait s'ensuivre, Frank l'accepta » (NS, p. 5) : il lie son destin à cet homme insignifiant qui ne le trahira pas et à sa fille Sissy qui continuera à l'aimer malgré tout ce qu'il lui fera endurer. Parti 
d'un simple jeu de provocation, un mécanisme implacable se met en route : jusqu'où Frank devra-t-il aller ? «Car il voulait que le destin s'occupât de lui ; il avait tout fait pour l'y forcer, il continuait à le défier du matin au soir» (NS, p. 138). Comme dans un contreapprentissage, il se salit et s'abîme, prend des risques, refuse de fuir, jusqu'à son arrestation, où il finit par rencontrer son destin tant attendu. Il est emprisonné dans une ancienne école, interrogé de longues heures à n'importe quel moment du jour et de la nuit. Violemment frappé au visage, il perd trois dents. Il se laisse pousser la barbe, porte des vêtements sales. Décidé à tenir jusqu'au bout, faisant preuve d'une grande force mentale, il accomplit son destin, c'est-àdire qu'il accepte totalement sa mort inévitable. Sur une toile de fond parfaitement réaliste, cet apprentissage ne se fait pas sans quelques métaphores et métamorphoses. Premièrement, Frank est enfermé dans une ancienne école : «ce n'est pas dans une prison qu'on l'a mis, mais dans une école, et cela doit avoir un sens » (NS, p. 163). Frank expérimente donc son emprisonnement comme une initiation (NS, p. 153), une voie à suivre, un «tunnel » duquel sortir (NS, p. 186). Pour tenir, il apprend à dormir, ou plutôt, ses geôliers le lui apprennent, à leur insu (NS, p. 202), ainsi que la patience et la faiblesse (NS, p. 230). Dans une logique de mortification, il accepte de souffrir pour ce qu'il a fait (NS, p. 194), les références religieuses sont d'ailleurs nombreuses : les hommes qui le surveillent quand il est interrogé sont des enfants de chœur (NS, pp. 206, 234), et celui qui l'interroge est une «bénédiction du ciel» $(N S$, p. 214). Son visage est « transfiguré $^{1} »$ quand il apprend que M. Hoslt a fait une demande de visite (NS, p. 232). Face à l'inéluctabilité de sa mort, Frank se hisse à un niveau de connaissance et de compréhension presque absolu. Pour cela, il doit entrer dans un état second : «il pense surtout par images, par sensations » $(N S$, p. 212), il rêve beaucoup, la frontière entre le rêve et la réalité s'amenuise (NS, pp. 213, 225). Preuves qu'il est dans un état second, les métamorphoses qui s'opèrent dans son

\footnotetext{
${ }^{1}$ On ne peut s'empêcher de penser au visage du prêtre Pierre Harmignie, défiguré par Christian Simenon lors de la tuerie de Courcelles.
} 
environnement: son interrogateur sadique et minutieux est un vieux monsieur avec qui il «discute» $(N S$, p. 213), sa cellule devient sa chambre et même son «chez lui » (NS, p. 233). Il parvient ainsi à reprendre en main le sens de sa vie, c'est-à-dire qu'il est capable de décider l'heure de sa mort: "sa seule crainte, sa hantise, c'est que cela se produise trop vite, avant l'heure qu'il a décidée, avant qu'il ait fini » $(N S$, p. 215). Que doit-il finir ? Il doit voir Holst, recevoir sa compréhension et son absolution, le reconnaitre comme père (NS, pp. 246-247). Il doit entendre Sissy lui dire qu'elle l'aime encore. S'opère alors une fusion : il se met à ressembler à Holst : sa barbe $(N S, \mathrm{p}$. 172), sa boîte de fer blanc (223), sa voix (250). La femme à la fenêtre qui s'affaire quotidiennement et que Frank observe chaque jour à la même heure, lui procurant une joie inégalée, c'est Sissy (NS, p. 251). Réconcilié avec lui-même, réconcilié avec les autres par l'intermédiaire de Holst et Sissy, ayant trouvé un père, une sœur et une femme, il peut alors aller à la mort, le col de son veston relevé, enfin comme tous les autres, comme ceux qu'il méprisait jadis.

\section{Conclusion \\ Dans Warna, la mort est intériorisée par l'intermédiaire de la neige qui métaphorise la guerre, c'est-à-dire la perte, tandis que dans La neige, la mort étant l'horizon, la neige permet de conjurer la saleté de la guerre et d'amener Frank au bout de son destin. La métamorphose de la réalité est opérée sous l'action de la neige qui permet une intériorisation et une dilation des faits historiques. Sans être un déni, ce phénomène n'en est pas moins symptomatique des écrivains d'un pays qui ne maîtrise pas son destin historique "comme s'il était décidé ailleurs" (Willems 1984b: 206). Chez Simenon, cette impuissance se traduit en une véritable crainte de l'histoire, perçue comme le «terrain de l'angoisse, de la division et du conflit» (Assouline 1992 : 409). Or, c'est justement cette position qui permet à nos deux auteurs de faire œuvre littéraire car :}


Qu'est-ce qu'une œuvre d'art sinon, non pas comme on l'a dit avec complaisance, une œuvre hors du temps, mais une œuvre dans le temps et qui ne cesse de le défier ? Une œuvre susceptible, contrairement à l'homme, de ressusciter ?

\section{Bibliographie}

Assouline, P. (1992) : Simenon. Paris : Gallimard.

Bachelard, G. (1942): L'eau et les rêves. Essai sur l'imagination de la matière. Paris : Librairie José Corti, 1991.

Deman, T. (1988) : Warna ou le poids de la neige : un rêve d'ailleurs. Textyles, en ligne, http://textyles.revues.org/1714 (consulté le 20 novembre 2017).

Ginette, M. (1988) : Genres et écriture dans Warna ou le poids de la neige. Textyles, en ligne, http://textyles.revues.org/1716 (consulté le 20 novembre 2017).

Quaghebeur, M. (2017) : Du fantastique réel au réalisme magique ou d'une guerre mondiale à l'autre. Tout est réel ici de Paul Willems. In : R. Bizek-Tatara (dir.), Au-delà du réel. Écritures du surnaturel dans les lettres belges francophones. Lublin : Wydawnictwo Uniwersytetu Marii Curie-Skłodowskiej, pp. 15-44.

Simenon, G. (1948) : La Neige était sale. Paris : Presses de la cité, 1951.

Willems, P. (1974) : Les Miroirs d'Ostende. Bruxelles : Jacques Antoine.

Willems, P. (1984a) : Warna ou le poids de la neige. Bruxelles : Didascalies.

Willems, P. (1984b), Le Monde de Paul Willems. Textes, études, documents. Rassemblés par P. Emond, H. Ronse et F. Van de Kerckhove. Bruxelles : Éditions Labor, 1992.

${ }^{2}$ Compère, G. (1992) : Lettre à Paul Emond. In : Le Monde de Paul Willems. Textes, études, documents. Rassemblés par Emond P., Ronse H. et Van de Kerckhove F. Bruxelles : Éditions Labor, p.178. 